Proc. Indian Acad. Sci. (Chem. Sci.), Vol. 105, No. 6, December 1993, pp. 575-581.

(C) Printed in India.

\title{
Preparation of some organic precursors via photolytic demetallation of their iron complexes
}

\author{
ALAA S ABD-EL-AZIZ*, CHRISTINE R DE DENUS and \\ KRYSTYNA LEZYNSKA \\ Department of Chemistry, University of Winnipeg, Winnipeg, Manitoba, R3B 2E9, Canada
}

\begin{abstract}
Nucleophilic aromatic substitution $\left(S_{N} \mathrm{Ar}\right)$ of chloroarenes complexed to the cyclopentadienyl iron moiety with ethyl cyanoacetate or phenylsulfonylacetonitrile in the presence of potassium carbonate in DMF resulted in the formation of arylated ethyl cyanoacetate or phenylsulfonylacetonitrile complexes. Photolytic demetallation of these complexes led to the liberation of the substituted arene ligands in high yield. This synthetic route is more advantageous than those previously reported and is a practical way to synthesise heterocycles and alkanoic acid precursors.
\end{abstract}

Keywords. $S_{N}$ Ar; photolysis; ethyl arylcyanoacetate; aryl phenylsulfonylacetonitrile.

\section{Introduction}

There has been considerable interest in the development of new and efficient routes to the synthesis of arylated ethyl cyanoacetates and phenylsulfonylacetonitriles (Shen 1972; Suzuki et al 1987; Sakamoto et al 1990). These materials are known to be versatile intermediates in the synthesis of heterocyclic compounds (e.g. azetinones, pyrimidines, and oxazaphosphorinane derivatives) and alkanoic acids, which are used for medicinal purposes (Matsui and Motoi 1973; Giordano et al 1984; Shih and Wang 1984). One of the well-recognized alkanoic acids is 2-(4-isobutylphenyl)propanoic acid, known as Ibuprofen. This acid is an anti-inflammatory analgesic, finding use in the treatment of patients with rheumatoid arthritis and in relieving general muscle pain and stiffness (Adams et al 1969; Chalmers 1969; Shen 1979). The synthesis of ethyl arylcyanoacetates and arylated phenylsulfonylacetonitriles cannot be achieved directly through nucleophilic substitution reactions of ethyl cyanoacetate or phenylsulfonylacetonitrile anions with arylhalides. The routes to these compounds involve the use of certain organometallic reagents or catalysts to promote nucleophilic substitution on the aromatic ring (Kaiser et al 1971; Osuka et al 1983; Uno et al 1985; Sakamoto et al 1988; Kozyrod et al 1991). In these synthetic routes, the most problematic step is the nucleophilic aromatic substitution.

Activation of normally unreactive chloroarenes towards nucleophilic aromatic substitution reactions $\left(S_{N} \mathrm{Ar}\right)$ by complexation to a metal moiety such as chromium tricarbonyl, manganese tricarbonyl or cyclopentadienyliron $(\mathrm{CpFe})$ have been

* For correspondence 
reported (Semmelhack 1976; Pearson et al 1986; Kerber 1991). Nesmeyanov et al (1967) were the first to establish the efficient displacement of the chlorine atom in $\eta^{6}$-chlorobenzene- $\eta^{5}$-cyclopentadienyliron complex cations by some oxygen, sulfur and nitrogen nucleophiles. We have been very active in utilizing this methodology in the synthesis of various functionalized aromatic compounds (Abd-El-Aziz et al 1988, 1993; Abd-El-Aziz and de Denus 1992, 1993).

Nesmeyanov et al (1970) examined the effect of irradiation of arene complexes. They found that the photolytic degradation of these complexes in acetonitrile solution resulted in liberation of the arene ligands with ferrocene and iron(II) salt. This photolytic degradation was proven to be greatly dependent on the nature of the solvent, with $\sigma$-donor ligands resulting in the greatest yields. Gill and Mann (1980, 1981, 1983) have also given detailed descriptions of the photoinduced replacement of the arene by one 6-electron or three 2-electron ligands.

In this article, we describe our approach to the arylation of ethyl cyanoacetate and phenylsulfonylacetonitrile via $S_{N}$ Ar followed by photolytic demetallation.

\section{Results and discussion}

$\eta^{6}$-Chloroarene- $\eta^{5}$-cyclopentadienyliron hexafluorophosphates (1a-d) were prepared via ligand exchange reactions involving ferrocene and chlorobenzene, $o-, m$ - or $p$ dichlorobenzene according to the method reported by Khand et al (1968). Reactions of the product complexes (1a-d) with phenylsulfonylacetonitrile or ethyl cyanoacetate in the presence of potassium carbonate in $\mathrm{N}, \mathrm{N}$-dimethylformamide, led to the formation of complexed arylated ethyl cyanoacetates $(2 \mathrm{a}-\mathrm{d})$ and phenylsulfonylacetonitriles $(\underline{3 a}-d)$ as shown in scheme 1 . These complexes were isolated as yellow solids or yellow-brown oils in very good yields (71-94\%). Ortho substituents on the complexed aromatic ring did not cause any sterically induced inhibition of the reaction. ${ }^{1} \mathrm{H}$ and ${ }^{13} \mathrm{C}$ NMR and IR were used to characterize the prepared complexes (2a-d, $\underline{3 a-d}$ ). In the ${ }^{1} \mathrm{H}$ NMR spectra of these complexes, a very distinctive singlet appeared around 5.31-5.47 ppm which is characteristic of the cyclopentadienyl (Cp) ring. For complexes ( $\underline{3 \mathrm{a}}-\mathrm{d})$, in many cases the methine proton appeared at 6-7 ppm overlapping with the arene protons. The assignment of some of these methine protons was based on the integration of the peaks in the region between 6-7 ppm relative to other proton peaks in the spectra. The ${ }^{13} \mathrm{C}$ NMR was also in agreement with expectations, as is outlined in $\S 3$.
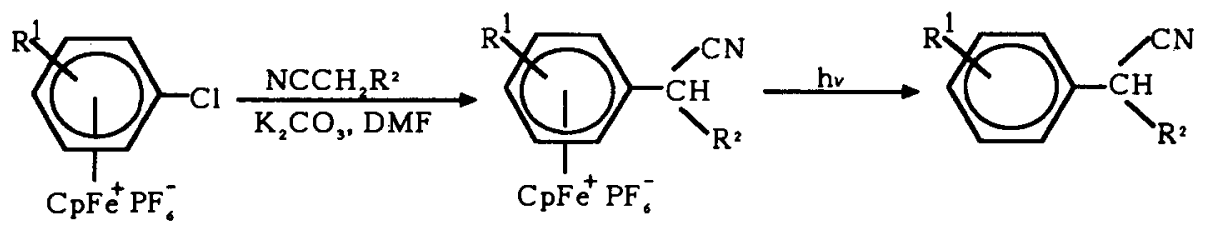

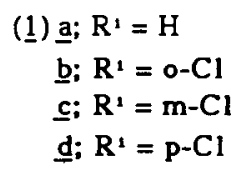

(2a-d) $R^{2}=$ COOEt

(4a- $-\underline{d}) R^{2}=$ COOEt

(ㅁa-d) $\mathrm{R}^{2}=\mathrm{SO}_{2} \mathrm{Ph}$

(ㅁa-d) $\mathrm{R}^{2}=\mathrm{SO}_{2} \mathrm{Ph}$

Scheme 1. 
Liberation of the arylated ethyl cyanoacetates and phenylsulfonylacetonitriles from their iron complexes is one of the most important steps in this synthetic strategy. Since photolysis is known to be an efficient method for the decomplexation of some (arene)cyclopentadienyliron complexes, we successfully applied this technique to liberate the free organic ligands. The complexes were dissolved in a mixture of acetonitrile/dichloromethane and irradiated under a nitrogen atmosphere for $2 \mathrm{~h}$, using a Xenon lamp as the source of radiation. Purification of the products by column chromatography resulted in isolation of the free aromatic ligands (4a- $\underline{\mathrm{d}}, \underline{5 \mathrm{a}}-\underline{\mathrm{d}})$ in yields ranging from $70-84 \%$.

The identities of all photolysed products were confirmed by ${ }^{1} \mathrm{H}$ and ${ }^{13} \mathrm{C} N \mathrm{NMR}$, IR, and MS. The major differences in the ${ }^{1} \mathrm{H}$ and ${ }^{13} \mathrm{C}$ NMR of these compounds from the complexes are the absence of the cyclopentadienyl peak, the shift of the arene peaks downfield, and a shift of the methine peak to a higher field. Compounds $(\underline{4 a}-d)$ are some of the heterocyclic precursors, and compounds $(\underline{5 a}-d)$ are the desired precursors for some alkanoic acid synthesis. Our synthetic methodology is extremely versatile and efficient in the preparation of such precursors.

In conclusion, we would like to point out that photolysis is an important and efficient step in the synthetic strategy and the $S_{N} A r$ route to the synthesis of arylated ethyl cyanoacetates and phenylsulfonylacetonitriles is very useful owing to its ease and the use of mild reagents.

\section{Experimental}

Ethyl cyanoacetate, phenylsulfonylacetonitrile, potassium carbonate and $\mathbf{N}, \mathbf{N}$ dimethylformamide (DMF) are commercially available and were used without further purification.

${ }^{1} \mathrm{H}$ and ${ }^{13} \mathrm{C}$ NMR were recorded on a Gemini 200 NMR spectrometer, with chemical shifts calculated from the solvent signals. MS spectra were recorded on a HP 5970 Series Mass Selective Detector in $m / z$ units. IR spectra were recorded using a Perkin-Elmer 78.1 spectrophotometer.

\subsection{Nucleophilic substitution reactions}

(a) Reactions with ethyl cyanoacetate: A mixture of $1 \mathrm{mmol}$ of starting cation (1a-d), $2.5 \mathrm{mmol}$ potassium carbonate, and $1.05 \mathrm{mmol}$ of ethyl cyanoacetate in $10 \mathrm{ml}$ of DMF was stirred at $50-60^{\circ} \mathrm{C}$, under a nitrogen atmosphere for $3 \mathrm{~h}$. The resulting dark red reaction mixture was filtered into $10 \mathrm{ml}$ of $10 \%$ hydrochloric acid. A concentrated aqueous solution of ammonium hexafluorophosphate was added to the reaction mixture, and the product extracted with dichloromethane $(3 \times 50 \mathrm{ml})$. The combined extract was washed with water $(4 \times 40 \mathrm{ml})$, dried over magnesium sulphate, and the solvent was evaporated off under reduced pressure at $25^{\circ} \mathrm{C}$. The residual yellow-brown oil was washed with diethyl ether $(3 \times 20 \mathrm{ml})$. Thus the following compounds were prepared.

(b) $\left(\eta^{5}\right.$-Cyclopentadienyl) [ $\eta^{6}$-ethyl 2-phenyl cyanoacetate]-iron(II) hexafluorophosphate (2a): $(0.362 \mathrm{~g}, 80 \%) v_{\max }\left(\mathrm{cm}^{-1}\right)$ (neat) $2260(\mathrm{CN}), 1755(\mathrm{CO}) ; \delta_{\mathrm{H}}\left(\mathrm{CD}_{3} \mathrm{COCD}_{3}\right)$ $1 \cdot 24\left(3 \mathrm{H}, t, J=7 \cdot 1, \mathrm{CH}_{3}\right), 4 \cdot 24\left(2 \mathrm{H}, q, J=6 \cdot 7, \mathrm{CH}_{2}\right), 5.31(5 \mathrm{H}, s, \mathrm{Cp}),(5 \cdot 72(1 \mathrm{H}, s$, $\left.\mathrm{CH}), 6.63(5 \mathrm{H}, b r, s, \mathrm{ArH}) ; \delta_{\mathrm{C}}\left(\mathrm{CD}_{3} \mathrm{COCD}\right)_{3}\right) 13.86\left(\mathrm{CH}_{3}\right), 43.11(\mathrm{CH}), 64.55\left(\mathrm{CH}_{2}\right)$, 
78.83 (5C, Cp), 86.98, 89.09, 89.19, 89.37, 90.21 (5C, ArC), 96.93 (quaternary ArC), $115 \cdot 33(\mathrm{CN}), 163 \cdot 70(\mathrm{CO})$.

(c) $\left(\eta^{5}\right.$-Cyclopentadienyl) [ $\eta^{6}$-ethyl-(o-chlorophenyl) cyanoacetate $]$-iron(II) hexafluorophosphate $(\underline{2 b}):(0.346 \mathrm{~g}, 71 \%) \quad v_{\max }\left(\mathrm{cm}^{-1}\right)$ (neat) 2305 (CN), 1755 (CO); $\delta_{\mathrm{H}}\left(\mathrm{CD}_{3} \mathrm{COCD}_{3}\right) 1.25\left(3 \mathrm{H}, t, J=7 \cdot 2, \mathrm{CH}_{3}\right), 4 \cdot 30\left(2 \mathrm{H}, q, J=6 \cdot 8, \mathrm{CH}_{2}\right), 5.47(5 \mathrm{H}, \mathrm{s}$, $\mathrm{Cp}), 6.26(1 \mathrm{H}, s, \mathrm{CH}), 6.71(1 \mathrm{H}, t, \mathrm{~J}=6.1, \mathrm{ArH}), 6.85(2 \mathrm{H}, m, \mathrm{ArH}), 7.05(1 \mathrm{H}, d, J=7.3$, $\mathrm{ArH}) ; \delta_{\mathrm{C}}\left(\mathrm{CD}_{3} \mathrm{COCD}_{3}\right)$ 13.76 $\left(\mathrm{CH}_{3}\right), 41 \cdot 56(\mathrm{CH}), 64.67\left(\mathrm{CH}_{2}\right), 80.87(5 \mathrm{C}, \mathrm{Cp}), 87 \cdot 12$, 88.53, 89.71, 89.90 (4C, ArC), 95.30, 108.56 (quaternary ArC), $114 \cdot 85$ (CN), $162 \cdot 48$ (CO). (d) ( $\eta^{5}$-Cyclopentadienyl) [ $\eta^{6}$-ethyl-(m-chlorophenyl) cyanoacetate]-iron $(I I)$ hexafluorophosphate $(\underline{2 c)}):(0.365 \mathrm{~g}, 74 \%) v_{\max }\left(\mathrm{cm}^{-1}\right)$ (neat) $2200(\mathrm{CN}), 1760(\mathrm{CO}) ; \delta_{\mathrm{H}}\left(\mathrm{CD}_{3}\right.$ $\left.\mathrm{COCD}_{3}\right) 1.26\left(3 \mathrm{H}, t, J=7 \cdot 1, \mathrm{CH}_{3}\right) 4.31\left(2 \mathrm{H}, q, J=7 \cdot 1, \mathrm{CH}_{2}\right), 5.46(5 \mathrm{H}, s, \mathrm{Cp}), 5.83$ $(1 \mathrm{H}, s, \mathrm{CH}), 6 \cdot 65-7.05(4 \mathrm{H}, t, J=6 \cdot 1, \mathrm{ArH}) ; \delta_{\mathrm{C}}\left(\mathrm{CD}_{3} \mathrm{COCD}_{3}\right) 13.02\left(\mathrm{CH}_{3}\right), 41 \cdot 74(\mathrm{CH})$, $63.93\left(\mathrm{CH}_{2}\right), 80.28(5 \mathrm{C}, \mathrm{Cp}), 86.41,87.98,88.96,89.82$ (4C, $\left.\mathrm{ArC}\right), 96.04,107.08$ (quaternary $\mathrm{ArC}), 115 \cdot 26(\mathrm{CN}), 162 \cdot 45(\mathrm{CO})$.

(e) ( $\eta^{5}$-Cyclopentadienyl) $\left[\eta^{6}\right.$-ethyl-(p-chlorophenyl) cyanoacetate $]$-iron $(I I)$ hexafluorophosphate $(2 d): \quad(0.396 \mathrm{~g}, 81 \%) v_{\max }\left(\mathrm{cm}^{-1}\right)$ (neat) $2310(\mathrm{CN}), 1760(\mathrm{CO}) ; \delta_{\mathrm{H}}\left(\mathrm{CD}_{3}\right.$ $\left.\mathrm{COCD}_{3}\right) 1.25\left(3 \mathrm{H}, t, J=7 \cdot 0, \mathrm{CH}_{3}\right), 4.25\left(2 \mathrm{H}, q, J=7 \cdot 1, \mathrm{CH}_{2}\right), 5.42(5 \mathrm{H}, s, \mathrm{Cp}), 5.72$ $(1 \mathrm{H}, s, \mathrm{CH}), 6.74(2 \mathrm{H}, d, J=5 \cdot 1, \mathrm{ArH}), 6.97(2 \mathrm{H}, d, J=6.5, \mathrm{ArH}) ; \delta_{\mathrm{C}}\left(\mathrm{CD}_{3} \mathrm{COCD}_{3}\right)$ $13.07\left(\mathrm{CH}_{3}\right), 41.67(\mathrm{CH}), 63.91\left(\mathrm{CH}_{2}\right), 80.41(5 \mathrm{C}, \mathrm{Cp}), 86 \cdot 11,88.52,88.55,88.64(4 \mathrm{C}$, $\mathrm{ArC}), 95 \cdot 34,107 \cdot 48$, (quaternary $\mathrm{ArC}$ ), $115 \cdot 30(\mathrm{CN}), 162 \cdot 54(\mathrm{CO})$.

\subsection{Reactions with phenylsulfonylacetonitrile}

A mixture of $1 \mathrm{mmol}$ of starting cation $(\underline{1 \mathrm{a}}-\mathrm{d}), 2.5 \mathrm{mmol}$ of potassium carbonate, $1.05 \mathrm{mmol}$ phenylsulfonylacetonitrile, and $10 \mathrm{ml}$ of DMF was stirred at room temperature under a nitrogen atmosphere for $\approx 7 \mathrm{~h}$. The resulting red reaction mixture was rapidly filtered into $10 \%$ hydrochloric acid $(10 \mathrm{ml})$. The reaction flask was then washed with ethanol and the washing added to the filtrate. The ethanol was removed under reduced pressure at $25^{\circ} \mathrm{C}$ using a rotary evaporator (Buchi RE-111). A concentrated aqueous solution of ammonium hexafluorophosphate was added to the reaction mixture. The solution was then stirred for $10 \mathrm{~min}$ and the resulting yellow product was collected by filtration. Thus the following were prepared.

(a) $\left(\eta^{5}\right.$-Cyclopentadienyl) [ $\eta^{6}$-phenyl phenylsulphonylacetonitrile]-iron(II) hexafluorophosphate $(\underline{3 a}):(0.414 \mathrm{~g}, 79 \%) v_{\max }\left(\mathrm{cm}^{-1}\right)$ (neat) $2320(\mathrm{CN}), 1335,1165$ (SO); $\delta_{\mathrm{H}}\left(\mathrm{CD}_{3} \mathrm{COCD}_{3}\right) 5.32(5 \mathrm{H}, s, \mathrm{Cp}), 6.46-6.70(6 \mathrm{H}, \mathrm{m}$, complexed $\mathrm{ArH}$ and $\mathrm{CH})$, 7.71-7.80 (2H, $\left.m, \mathrm{SO}_{2} \mathrm{PhH}\right), 7.85-7.99\left(3 \mathrm{H}, m, \mathrm{SO}_{2} \mathrm{PhH}\right) ; \delta_{\mathrm{C}}\left(\mathrm{CD}_{3} \mathrm{COCD}_{3}\right) 61.96$ $(\mathrm{CH}), 79 \cdot 41$ (5C, Cp), 87.68, 89.78, 89.82, 90.31, 91.47 (5C, ArC), $92 \cdot 20$ (quaternary $\mathrm{ArC}$ ), $113 \cdot 13(\mathrm{CN}), 130 \cdot 81\left(2 \mathrm{C}, \mathrm{SO}_{2} \mathrm{PhC}\right.$ ), 130.91 (2C, $\mathrm{SO}_{2} \mathrm{PhC}$ ), $137 \cdot 29$ (quaternary $\mathrm{SO}_{2} \mathrm{PhC}$ ).

(b) ( $\eta^{5}$-Cyclopentadienyl) $\quad\left[\eta^{6}-(o-c h l o r o p h e n y l)\right.$-phenylsulphonyl-acetonitrile $]$-iron(II) hexafluorophosphate $(\underline{3 b}): \quad(0.527 \mathrm{~g}, 94 \%) v_{\max }\left(\mathrm{cm}^{-1}\right)$ (neat) $2300(\mathrm{CN}), 1338,1163$ (SO). $\delta_{\mathrm{H}}\left(\mathrm{CD}_{3} \mathrm{COCD}_{3}\right) 5.46(5 \mathrm{H}, \mathrm{s}, \mathrm{Cp}), 6.71-7.08(5 \mathrm{H}, \mathrm{s}$, complexed $\mathrm{ArH}$ and $\mathrm{CH})$, 7.76-7.83 (2H, $\left.m, \mathrm{SO}_{2} \mathrm{PhH}\right), 7.93-8.02\left(3 \mathrm{H}, m, \mathrm{SO}_{2} \mathrm{PhH}\right) . \delta_{\mathrm{C}}\left(\mathrm{CD}_{3} \mathrm{COCD}_{3}\right) 60.25$ (CH), 81.86 (5C, Cp), 89.40 (quaternary $\mathrm{ArC}$ ), 88.10, 89.61, 90.96, 91.09 (4C, ArC), 109.50 (quaternary ArC), 113.41 (CN), 130.93 (2C, $\left.\mathrm{SO}_{2} \mathrm{PhC}\right), 131.23$ (2C, $\mathrm{SO}_{2} \mathrm{PhC}$ ), 135.41 (quaternary $\mathrm{SO}_{2} \mathrm{PhC}$ ), and $137.63\left(\mathrm{SO}_{2} \mathrm{PhC}\right)$.

(c) $\left(\eta^{5}\right.$-Cyclopentadienyl) [ $\left[\eta^{6}-(m-\right.$ chlorophenyl $)$-phenylsulphonyl-acetonitrile $]$-iron $(I I)$ hexafluorophosphate $(\underline{3 \mathrm{c}}):(0.451 \mathrm{~g}, 81 \%) v_{\max }\left(\mathrm{cm}^{-1}\right)$ (neat) $2280(\mathrm{CN}), 1345,1162$ 
$(\mathrm{SO}) ; \delta_{\mathrm{H}}\left(\mathrm{CD}_{3} \mathrm{COCD}_{3}\right) 5.47(5 \mathrm{H}, \mathrm{s}, \mathrm{Cp}), 6.40-7.06(5 \mathrm{H}, m$, complexed $\mathrm{ArH}$ and $\mathrm{CH})$, 7.75-7.85 (2H, $\left.m, \mathrm{SO}_{2} \mathrm{PhH}\right), 7.90-8.05\left(3 \mathrm{H}, m, \mathrm{SO}_{2} \mathrm{PhH}\right) ; \delta_{\mathrm{C}}\left(\mathrm{CD}_{3} \mathrm{COCD}_{3}\right) 61.38$ (CH), $81.77(5 \mathrm{C}, \mathrm{Cp}), 86.57,87.24,89 \cdot 61,90.85$ (4C, $\mathrm{ArC}), 92.95$ (quaternary $\mathrm{ArC}$ ), 108.44 (quaternary $\mathrm{ArC}$ ), $112.71(\mathrm{CN}), 130.93\left(4 \mathrm{C}, \mathrm{SO}_{2} \mathrm{PhC}\right.$ ), 135.17 (quaternary $\left.\mathrm{SO}_{2} \mathrm{PhC}\right), 137 \cdot 40\left(\mathrm{SO}_{2} \mathrm{PhC}\right)$.

(d) ( $\eta^{5}$-Cyclopentadienyl) $\left[\eta^{6}-(p\right.$-chlorophenyl)-phenylsulphonyl-acetonitrile $]$-iron $(I I)$ hexafluorophosphate $(\underline{3 d}):(0.428 \mathrm{~g}, 77 \%) v_{\max }\left(\mathrm{cm}^{-1}\right)$ (neat) $2305(\mathrm{CN}),(1348,1142$ (SO); $\delta_{\mathrm{H}}\left(\mathrm{CD}_{3} \mathrm{COCD}_{3}\right) 5.44(5 \mathrm{H}, \mathrm{s}, \mathrm{Cp}), 6.55-6.77(3 \mathrm{H}, \mathrm{m}$, complexed ArH and $\mathrm{CH})$, $7.02\left(2 \mathrm{H}, d, J=6 \cdot 2\right.$, complexed ArH), 7.72-7.81 (2H, $\left.m, \mathrm{SO}_{2} \mathrm{PhH}\right), 7 \cdot 89-7.98(3 \mathrm{H}, m$, $\left.\mathrm{SO}_{2} \mathrm{PhH}\right) ; \delta_{\mathrm{C}}\left(\mathrm{CD}_{3} \mathrm{COCD}_{3}\right) 61 \cdot 12(\mathrm{CH}), 81 \cdot 73(5 \mathrm{C}, \mathrm{Cp}), 87 \cdot 40,90 \cdot 04,90 \cdot 07,91 \cdot 15(4 \mathrm{C}$, $\mathrm{ArC}$ ), 91.51 (quaternary ArC), 109.09 (quaternary $\mathrm{ArC}$ ), $112.93(\mathrm{CN}), 130.80(2 \mathrm{C}$, $\mathrm{SO}_{2} \mathrm{PhC}$ ), $130 \cdot 91\left(2 \mathrm{C}, \mathrm{SO}_{2} \mathrm{PhC}\right.$ ), $134 \cdot 70$ (quaternary $\mathrm{SO}_{2} \mathrm{PhC}$ ), and $137 \cdot 32\left(\mathrm{SO}_{2} \mathrm{PhC}\right.$ ).

\section{Photolysis}

$1.0 \mathrm{mmol}$ of complexes $\underline{2 \mathrm{a}}-\underline{\mathrm{d}}, \underline{3 \mathrm{a}}-\underline{\mathrm{d}}$ were dissolved in a mixture of $\mathrm{CH}_{2} \mathrm{Cl}_{2} / \mathrm{CH}_{3} \mathrm{CN}$ $(30 \mathrm{ml}: 10 \mathrm{ml})$ in a pyrex tube. The solution was deoxygenated by bubbling nitrogen through it and the reaction tube was fitted into a photochemical apparatus equipped with a xenon lamp (lower limit $=290 \mathrm{~nm}$ ), and irradiated at room temperature for $2 \mathrm{~h}$. The solvent was concentrated to a volume of 1-2 $\mathrm{ml}$ using rotary evaporation, and introduced into a column of silica gel, prepared in hexane. The residue was washed with hexane, and the product eluted with chloroform by passage through a short column. Removal of the solvent from the eluate gave the expected liberated arene, with the following yields and spectral data.

(a) Ethyl 2-phenyl cyanoacetate ( $\underline{4 a})$ : Yellowish crystals $(0 \cdot 151 \mathrm{~g}, 80 \%) \cdot v_{\max }\left(\mathrm{cm}^{-1}\right)$ (neat) $2220(\mathrm{CN}), 1750(\mathrm{CO}) ; \delta_{\mathrm{H}}\left(\mathrm{CDCl}_{3}\right): 1 \cdot 26\left(3 \mathrm{H}, t, J=7 \cdot 1, \mathrm{CH}_{3}\right), 4 \cdot 23(2 \mathrm{H}, q, J=7 \cdot 2$, $\left.\mathrm{CH}_{2}\right), 4.70(1 \mathrm{H}, s, \mathrm{CH}), 7.38-7.46(5 \mathrm{H}, \mathrm{br} s, \mathrm{ArH}) . \delta_{\mathrm{C}}\left(\mathrm{CDCl}_{3}\right): 13.87\left(\mathrm{CH}_{3}\right), 43.76$ $(\mathrm{CH}), 63.29\left(\mathrm{CH}_{2}\right), 115.60(\mathrm{CN}), 123.05$ (quaternary ArC), 127.89, 129.20, $129.32(5 \mathrm{C}$, ArC), and 164.50 (CO). $m / z 189\left(M^{+}, 3 \%\right), 145(5), 117(100), 89(24)$.

(b) Ethyl-(o-chlorophenyl) cyanoacetate $(\underline{4 b}): \quad(0.183 \mathrm{~g}, 82 \%)$, yellowish oil. $v_{\max }\left(\mathrm{cm}^{-1}\right)$ (neat) $2220(\mathrm{CN}), 1750(\mathrm{CO}) ; \delta_{\mathrm{H}}\left(\mathrm{CDCl}_{3}\right): 1 \cdot 26\left(3 \mathrm{H}, t, J=7 \cdot 1, \mathrm{CH}_{3}\right), 4 \cdot 23(2 \mathrm{H}, q, J=7 \cdot 2$, $\left.\mathrm{CH}_{2}\right), 4.70(1 \mathrm{H}, s, \mathrm{CH}), 7 \cdot 38-7.46(5 \mathrm{H}, b r, s, \mathrm{ArH}) . \delta_{\mathrm{C}}\left(\mathrm{CDCl}_{3}\right): 13.87\left(\mathrm{CH}_{3}\right), 43.76$ $(\mathrm{CH}), 63.29\left(\mathrm{CH}_{2}\right), 115.60(\mathrm{CN}), 123.05$ (quaternary $\left.\mathrm{ArC}\right), 127 \cdot 89,129 \cdot 20,129.32(5 \mathrm{C}$ : ArC), and $164 \cdot 50(\mathrm{CO}) . \mathrm{m} / z 225\left[\left({ }^{37} \mathrm{Cl}\right), 2\right], 223\left[\left({ }^{35} \mathrm{Cl}\right), 5\right], 151(100), 116(20)$.

(c) Ethyl-(m-chlorophenyl) cyanoacetate $\left(\underline{4 c)}: \quad(0.187 \mathrm{~g}, 84 \%)\right.$, yellowish oil. $v_{\max }\left(\mathrm{cm}^{-1}\right)$ (neat) $2260(\mathrm{CN}), 1755(\mathrm{CO}) ; \delta_{\mathrm{H}}\left(\mathrm{CDCl}_{3}\right): 1 \cdot 27\left(3 \mathrm{H}, t, J=7 \cdot 0, \mathrm{CH}_{3}\right), 4 \cdot 25(2 \mathrm{H}, q, J=7 \cdot 1$ $\left.\mathrm{CH}_{2}\right), 4.67(1 \mathrm{H}, s, \mathrm{CH}), 7.35(4 \mathrm{H}, b r s, \mathrm{ArH}) . \delta_{\mathrm{C}}\left(\mathrm{CDCl}_{3}\right): 13.82\left(\mathrm{CH}_{3}\right), 43.20(\mathrm{CH})$ $63.56\left(\mathrm{CH}_{2}\right), 115.23(\mathrm{CN}), 126.08,128.09,129.48,130.51(4 \mathrm{C}, \mathrm{ArC}), 131.59,135.15$ (quaternary $\mathrm{ArC}$ ) and $164 \cdot 34(\mathrm{CO}) . \mathrm{m} / z 225\left[\left({ }^{37} \mathrm{Cl}\right), 4\right], 223\left[\left({ }^{35} \mathrm{Cl}\right), 11\right], 151(100)$ 116 (32).

(d) Ethyl-(p-chlorophenyl) cyanoacetate $(\underline{4 d}): \quad(0.176 \mathrm{~g}, 79 \%)$, yellowish oil. $v_{\max }\left(\mathrm{cm}^{-1}\right.$ (neat) $2300(\mathrm{CN}), 1750(\mathrm{CO}) ; \delta_{\mathrm{H}}\left(\mathrm{CDCl}_{3}\right): 1 \cdot 26\left(3 \mathrm{H}, t, J=7 \cdot 1, \mathrm{CH}_{3}\right), 4 \cdot 23(2 \mathrm{H}, q, J=7 \cdot 1$ $\left.\mathrm{CH}_{2}\right), 4.68(1 \mathrm{H}, \mathrm{s}, \mathrm{CH}), 7.39(4 \mathrm{H}, \mathrm{br} s, \mathrm{ArH}) . \delta_{\mathrm{C}}\left(\mathrm{CDCl}_{3}\right): 13.82\left(\mathrm{CH}_{3}\right), 43.05(\mathrm{CH})$ $63.49\left(\mathrm{CH}_{2}\right), 115.22(\mathrm{CN}), 128.37$ (quaternary $\left.\mathrm{ArC}\right), 129 \cdot 25,129.49(4 \mathrm{C}, \mathrm{ArC}), 135.4($ (quaternary ArC) and 164.52(CO). $\mathrm{m} / \mathrm{z} 225\left[\left({ }^{37} \mathrm{Cl}\right), 3\right], 223\left[\left({ }^{35} \mathrm{Cl}\right), 10\right], 151(97), 116(15)$. (e) Phenyl phenylsulphonylacetonitrile ( $(5 \mathrm{a})$ : Yellowish solid $(0.206 \mathrm{~g}, 80 \%) \cdot v_{\max }\left(\mathrm{cm}^{-1}\right.$ (neat) $2310(\mathrm{CN}), 1370,1160(\mathrm{SO}) . \delta_{\mathrm{H}}\left(\mathrm{CDCl}_{3}\right): 5 \cdot 15(1 \mathrm{H}, \mathrm{s}, \mathrm{CH})$, and $7 \cdot 25-7 \cdot 74(10 \mathrm{H}$ 
$m, 2 \mathrm{Ph}) . \delta_{\mathrm{C}}\left(\mathrm{CDCl}_{3}\right): 63.00(\mathrm{CH}), 113.37(\mathrm{CN}), 125.34$ (quaternary $\left.\mathrm{ArC}\right), 128.98(2 \mathrm{C}$, $\left.\mathrm{SO}_{2} \mathrm{PhC}\right), 129 \cdot 14$ (2C, $\left.\mathrm{SO}_{2} \mathrm{PhC}\right), 129.68$ (2C, $\left.\mathrm{ArC}\right), 130.01$ (2C, $\left.\mathrm{ArC}\right), 130.45$ (1C, ArC), $134 \cdot 29$ (quaternary $\mathrm{SO}_{2} \mathrm{PhC}$ ), and $135 \cdot 19\left(\mathrm{SO}_{2} \mathrm{PhC}\right) . m / z 257\left(M^{+}, 15 \%\right), 141$ (16), 116 (100), 77 (26).

(f) (o-Chlorophenyl)phenylsulphonylacetonitrile ( $\underline{5 b})$ : White solid $(0.215 \mathrm{~g}, 74 \%)$. $v_{\max }\left(\mathrm{cm}^{-1}\right)$ (neat) $2305(\mathrm{CN}), 1340,1165(\mathrm{SO}) . \delta_{\mathrm{H}}\left(\mathrm{CDCl}_{3}\right): 5.81(1 \mathrm{H}, \mathrm{s}, \mathrm{CH}), 7 \cdot 34-7.63$ (4H, $m, \mathrm{ArH}), 7 \cdot 74-7 \cdot 85$ (2H, $\left.m, \mathrm{SO}_{2} \mathrm{PhH}\right), 7 \cdot 84-7.89$ (3H, $\left.m, \mathrm{SO}_{2} \mathrm{PhH}\right) . \delta_{\mathrm{C}}\left(\mathrm{CDCl}_{3}\right)$ : $58.94(\mathrm{CH}), 113.26(\mathrm{CN}), 124.08$ (quaternary $\mathrm{ArC}$ ), $127.70(\mathrm{ArC}), 129.50$ (2C, $\mathrm{SO}_{2} \mathrm{PhC}$ ), $130.00\left(2 \mathrm{C}, \mathrm{SO}_{2} \mathrm{PhC}\right), 130 \cdot 19,131 \cdot 26$, and 132.04 (3C, $\left.\mathrm{ArC}\right), 135.30$ (quaternary $\left.\mathrm{SO}_{2} \mathrm{PhC}\right), 135 \cdot 45\left(\mathrm{SO}_{2} \mathrm{PhC}\right), 135 \cdot 60$ (quaternary $\mathrm{ArC}$ ). $\mathrm{m} / z 293 \cdot\left[\left({ }^{37} \mathrm{Cl}\right), 8\right], 291\left[\left({ }^{35} \mathrm{Cl}\right)\right.$, 20], 150 (100), 77 (74).

(g) (m-Chlorophenyl)phenylsulphonylacetonitrile (5c): Yellowish oil $(0 \cdot 204 \mathrm{~g}, 70 \%)$. $v_{\max }\left(\mathrm{cm}^{-1}\right)$ (neat) $2305(\mathrm{CN})$ and $1340,1162(\mathrm{SO}) . \delta_{\mathrm{H}}\left(\mathrm{CDCl}_{3}\right): 5 \cdot 12(1 \mathrm{H}, \mathrm{s}, \mathrm{CH})$ and 7.18-7.46 (4H, $m, \mathrm{ArH}), 7 \cdot 52-7.62\left(2 \mathrm{H}, m, \mathrm{SO}_{2} \mathrm{PhH}\right), 7 \cdot 72-7.80\left(3 \mathrm{H}, m, \mathrm{SO}_{2} \mathrm{PhH}\right)$. $\delta_{\mathrm{C}}\left(\mathrm{CDCl}_{3}\right): 62 \cdot 16(\mathrm{CH}), 112.90(\mathrm{CN}), 127.09$ (quaternary $\left.\mathrm{ArC}\right), 127.82(\mathrm{ArC}), 129.22$ (2C, $\mathrm{SO}_{2} \mathrm{PhC}$ ), 129.62 (ArC), $129 \cdot 87$ (2C, $\mathrm{SO}_{2} \mathrm{PhC}$ ), 130.14, 130.60 (2C, $\left.\mathrm{ArC}\right), 133.99$ (quaternary $\mathrm{SO}_{2} \mathrm{PhC}$ ), 134.84 (quaternary $\mathrm{ArC}$ ), and $135.40\left(\mathrm{SO}_{2} \mathrm{PhC}\right) . \mathrm{m} / \mathrm{z} 293$ $\left[\left({ }^{37} \mathrm{Cl}\right), 4\right], 291\left[\left({ }^{35} \mathrm{Cl}\right), 14\right], 141(91), 77(100)$.

(h) (p-Chlorophenyl)phenylsulphonylacetonitrile (5d): Yellowish oil $(0.218 \mathrm{~g}, 75 \%)$. $v_{\max }\left(\mathrm{cm}^{-1}\right)$ (neat) $2305(\mathrm{CN})$ and 1338, $1160(\mathrm{SO}) . \delta_{\mathrm{H}}\left(\mathrm{CDCl}_{3}\right): 5 \cdot 12(1 \mathrm{H}, \mathrm{s}, \mathrm{CH})$, and $7.21(2 \mathrm{H}, d, J=8.4, \mathrm{ArH}), 7.34$ (2H, $d, J=8.4, \mathrm{ArH}), 7.51-7.65$ (2H, $\left.m, \mathrm{SO}_{2} \mathrm{PhH}\right)$, 7.75-7.81 (3H, $\left.m, \mathrm{SO}_{2} \mathrm{PhH}\right) . \delta_{\mathrm{C}}\left(\mathrm{CDCl}_{3}\right): 60.08(\mathrm{CH}), 113.07(\mathrm{CN}), 123.82$ (quaternary $\mathrm{ArC}$ ), $129 \cdot 25$ (4C, $\mathrm{SO}_{2} \mathrm{PhC}$ ), 129.91, 130.94 (4C, $\left.\mathrm{ArC}\right), 134 \cdot 13$ (quaternary $\mathrm{SO}_{2} \mathrm{PhC}$ ), 135.35 (SO ${ }_{2} \mathrm{PhC}$ ), and 136.90 (quaternary $\left.\mathrm{ArC}\right) \cdot \mathrm{m} / \mathrm{z} 293\left[\left({ }^{37} \mathrm{Cl}\right), 3\right], 291\left[\left({ }^{35} \mathrm{Cl}\right), 7\right]$, 150 (100), 141 (11), 77 (28).

\section{Acknowledgement}

Financial support by the University of Winnipeg is gratefully acknowledged.

\section{References}

Abd-El-Aziz A S and de Denus C R 1992 Synth. Commun. 22581

Abd-El-Aziz A S and de Denus C R $1993 \mathrm{~J}$. Chem. Soc., Perkin Trans. (accepted)

Abd-El-Aziz A S, Lee C C, Piorko A and Sutherland R G 1988a Synth. Commun. 18291

Abd-El-Aziz A S, Lee C C, Piorko A and Sutherland R G 1988b J. Organomet. Chem. 34895

Abd-El-Aziz A S, Tesfalidet S, de Denus C R and Lezynska K 1993 Synth. Commun. (accepted)

Adams S S, McCullough K F and Nicholson J S 1969 Arch. Int. Pharmacodyn. 178115

Chalmers T M 1969 Ann. Rheum. Dis. 28513

Gill T P and Mann K R 1980 Inorg. Chem. 193007

Gill T P and Mann K R 1981 J. Organomet. Chem. 221986

Gill T P and Mann K R 1983 Inorg. Chem. 221986

Giordano C, Castaldi G and Uggeri F 1984 Angew. Chem., Int. Ed. Engl. 23413

Kaiser E M, Solter L E, Schwarz R A, Beard R D and Hauser C R 1971 J. Am. Chem. Soc. 934237

Kerber R C 1991 J. Organomet. Chem. 404107

Khand I U, Pauson P L and Watts W F $1968 \mathrm{~J}$. Chem. Soc. (C) 2261

Kozyrod R P, Morgan J and Pinhey J T 1991 Aust. J. Chem. 44369

Matsui K and Motoi M 1973 Bull. Chem. Soc. Jpn. 461755

Nesmeyanov A N, Vol'kenau N A and Bolesova I N 1967 Dolk. Akad. Nauk. SSSR 175606 
Nesmeyanov A N, Vol'kenau N A and Shilovtseva L S 1970 Dokl. Nauk. Akad. SSSR 190857

Osuka A, Kobayashi T and Suzuki H 1983 Synthesis 67

Pearson A J, Bruhn P R and Hsu S-Y 1986 J. Org. Chem. 512137

Sakamoto T, Katoh E, Kondo Y and Yamanka H 1988 Chem. Pharm. Bull. 361664

Sakamoto T, Katoh E, Kondo Y and Yamanka H 1990 Chem. Pharm. Bull. 381513

Semmelhack M F 1976 J. Organomet. Chem. Libr. 1361

Shen T Y 1972 Angew. Chem., Int. Ed. Engl. 116

Shen T Y 1979 Prostaglandin synthetase inhibitors I. In Handbook of experimental pharmacology (Berlin Heidelberg, New York: Springer-Verlag) vol. 50/II, pp. 305-347

Shih Y and Wang J 1984 Heterocycles 222799

Suzuki H, Yi Q, Inoue J, Kusume K and Ogawa T 1987 Chem. Lett. 887

Uno M, Seto K, Ueda W, Masuda M and Takahashi S 1985 Synthesis 506 